\title{
Visual maps in the adult primate cerebral cortex: some implications for brain development and evolution
}

M.G.P. Rosa
Correspondence

M.G.P. Rosa

Department of Physiology

Monash University

Victoria 3800

Australia

Fax: +61-3-9905-2547

E-mail:

marcello.rosa@med.monash.edu.au

Presented at the IV International UNESCO Course on "What the

Developing Cerebral Cortex Tells About the Adult Cortex (and Vice Versa)", Rio de Janeiro, RJ, Brazil, December 3-7, 2001.
Research supported by the Australian Research Council and the National Health and Medical Research Council.

Received July 12, 2002 Accepted September 17, 2002
Department of Physiology, Monash University, Victoria, Australia

\begin{abstract}
In this paper, the topology of cortical visuotopic maps in adult primates is reviewed, with emphasis on recent studies. The observed visuotopic organisation can be summarised with reference to two basic rules. First, adjacent radial columns in the cortex represent partially overlapping regions of the visual field, irrespective of whether these columns are part of the same or different cortical areas. This primary rule is seldom, if ever, violated. Second, adjacent regions of the visual field tend to be represented in adjacent radial columns of a same area. This rule is not as rigid as the first, as many cortical areas form discontinuous, second-order representations of the visual field. A developmental model based on these physiological observations, and on comparative studies of cortical organisation, is then proposed, in order to explain how a combination of molecular specification steps and activity-driven processes can generate the variety of visuotopic organisations observed in adult cortex.
\end{abstract}

Key words

- Primate

- Vision

- Receptive fields

- Cortical areas

- Development

- Specification

.... Sur.

\section{Introduction}

In mammals, the anatomical connections subserving vision are characterised by precise patterns of origins and terminations. This anatomical specificity is such that information coming from topographically corresponding points of the two retinae converges onto single cells in the primary visual area (V1), and cells in adjacent regions of the same retina project to adjacent points of $\mathrm{V} 1$. As a result, cells in adjacent V1 columns always have receptive fields that represent slightly different, but overlapping portions of the visual field (1). This orderly relationship creates a visuotopic map, whereby V1 neurones on one side of the brain collec- tively represent the entire contralateral half of the visual field in a gradual and predictable fashion $(2,3)$.

There are, in addition to V1, many other visual areas, which collectively form the extrastriate cortex. Each extrastriate area has been traditionally viewed as forming a separate, more or less complete visuotopic map (e.g., 4,5). Thus, visuotopic organisation has become one of the key criteria for the subdivision of cortex: charting the various rerepresentations of the visual field should, in theory, be sufficient to define the limits of the various visual areas. However, it has gradually become clear that the relationship between areas and visuotopic maps is not that straightforward. For example, of the many 
extrastriate areas described by the pioneering mapping studies in monkeys, only two have become universally accepted: the second (V2), and middle temporal (MT) areas. Other cortical fields that were initially described as forming equally well-organised visuotopic maps have since been further subdivided, or had parts recombined with adjacent cortex to form "new" areas, which continue to be the subject of much controversy $(6,7)$. This uncertainty reflects not only the conflicting views of researchers regarding what constitutes a "cortical area", but also the development of our understanding of cortical areas. We are now aware of more subtle and complex types of organisation than originally envisaged, which translate into important caveats that must be considered when one interprets the way in which visuotopic maps relate to cortical areas. In this paper I will review some characteristics of cortical visuotopic maps in adults, and the implications that they may have for our understanding of the development and evolution of the cerebral cortex.

The numerous interactions between excitatory and inhibitory cells at each stage of visual processing result in cortical maps which depart in several ways from being precise point-to-point replicates of the sensory receptor surface. First, cortical maps are probabilistic: only the average receptive field position varies as a function of position in the cortex. Moreover, in many cases they include locally repetitive, mosaic-like representations of the same points of the visual field in adjacent columns, by cells with different response properties and connections. Thus, even the smallest stimulus in the visual field will evoke a response from an intricately shaped cluster of thousands of cortical cells distributed over a mean position. Second, cortical maps do not simply reflect the receptor densities at the periphery, and visuotopic maps can emphasise different parts of the visual field to different extents. For example, the central visual field is even more highly magnified in V1 than would be expected on the basis of ganglion cell densities alone (8); this emphasis on foveal representation can be more or less marked in different areas $(9,10)$. Finally, as detailed in the following section, the maps found in different cortical areas represent the contralateral visual hemifield according to different topological rules.

\section{Categories of visuotopic maps}

Given that the sets of information coming from the left and right halves of the visual field each project to the contralateral hemisphere, there are no truly continuous representations in the cortex. Nonetheless, if one restricts the analysis to the geometrical relationships between one hemifield and its contralateral cortical projection, different categories of geometrical transformation can be recognised on the basis of topological continuity. One significant distinction that can be made is between first- and secondorder transformations (or representations) of the visual field (4). First-order representations are those in which adjacent points of the same hemifield always map to adjacent columns in the contralateral cortex. One example of this type of transformation is the visuotopic map in V1. This can be demonstrated by considering the visual field locations indicated by points a-d, in Figure 1. As with any systematic progression of points in the same hemifield, the arc a-d maps onto a continuous series of cortical columns in V1 (Figure 1B). In this case, the surface being mapped (the visual hemifield) and the surface where the map exists (the cortical area) are referred to as topologically equivalent. Only two areas in the primate cortex form first-order representations of the visual field: V1 and MT (3,10-12). In contrast, secondorder representations are those in which the topological equivalence between the visual field and the cortex is disrupted. One example of a second-order map is found in V2 
(Figure 1B), where the upper and lower quadrants of the contralateral hemifield are represented in separate locations, being connected only by a small bridge corresponding to the foveal representation $(4,13)$. As a result, although the representations of the upper and lower contralateral quadrants in V2 are internally continuous (e.g., points a and b, or c and d), the representations of points above or below the horizontal meridian ${ }^{1}$ are nonadjacent in the cortex (points $\mathrm{b}$ and $\mathrm{c}$ ).

The map in V2 illustrates a type of second-order representation known as a field discontinuity map. These maps are organised as though a discontinuity had been introduced in the visual field or retina. The topology of this type of map can be considered by imagining that a first-order transformation has been cut along a line, such as the representation of the horizontal meridian, and physically parted (Figure 2). In the simian primates which have been studied to date, the line of field discontinuity in the V2 map corresponds to the horizontal meridian $(4,9$, 14,15). However, this is not necessarily the case, and in other animals, including prosimians, field discontinuities corresponding to different parts of the visual field have been reported $(16,17)$. It was initially suggested that second-order transformations including a single field discontinuity (usually coinciding with the representation of the horizontal meridian) were common in primate extrastriate cortex (e.g., 5, 18-20). However, it is now evident that extrastriate visuotopic maps can display more topological complexity than is apparent in V2, as well as more intra- and inter-specific variability. This complexity is expressed in at least two ways: multiple field discontinuities can exist in the same visuotopic map, and these may also be combined with another type of non-topologically equivalent transformation, the map discontinuity.

A good example of a complex pattern of field discontinuities is found in the third visual area (V3). V3 encompasses a large proportion of the cortical belt located imme-

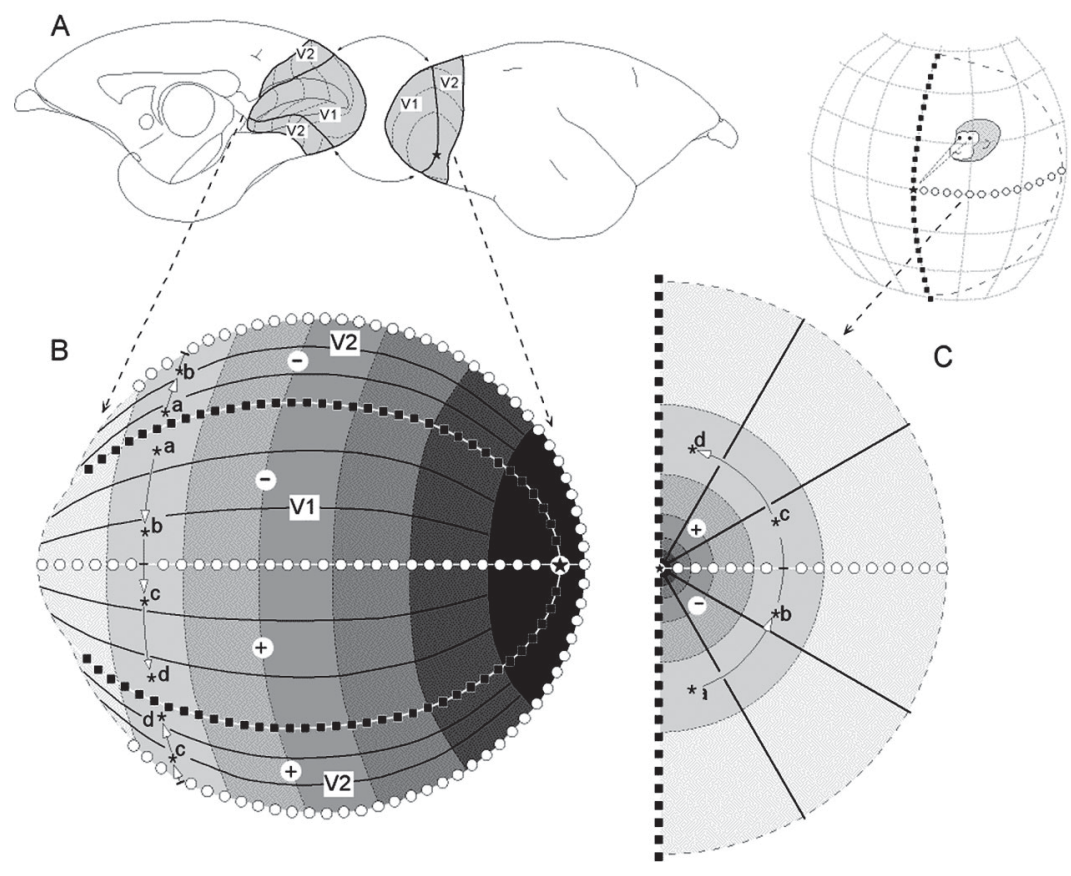

Figure 1. Visuotopic organisation of areas V1 and V2. A, Medial and lateral views of the right cerebral hemisphere of a marmoset monkey, indicating the locations of $\mathrm{V} 1$ and $\mathrm{V} 2$. In the medial view (top left) the banks of the calcarine sulcus have been separated to reveal portions of these areas. The star indicates the representation of the centre of the foveola, and isoeccentricity contours are indicated by fine dotted lines. The topological relationship between $\mathrm{V} 1$ and $\mathrm{V} 2$ on the medial and lateral surfaces is indicated by the arrows. $B, A$ schematic unfolded representation of $\mathrm{V} 1$ and $\mathrm{V} 2$ indicating the locations of the representations of different parts of the visual field. In this diagram, different visual field locations are indicated by different grey shades and symbols, which are summarised in a schematic view of the animal's contralateral hemifield (C). The representation of the central $1^{\circ}$ of the visual field is indicated in black, and progressively more eccentric parts of the visual field in gradually lighter shades of grey. Representations of the vertical meridian are outlined by the black squares, representations of the horizontal meridian by white circles, and those of the temporal perimeter of the visual field by dashed lines. The "+" and "-" signs indicate representations of the upper and lower contralateral quadrant, respectively. C, Schematic representation of the animal's left hemifield, with four points located along an isoeccentric $\operatorname{arc}(\mathrm{a}-\mathrm{d})$ indicated. Note that while the representation of this arc in V1 is continuous (B), in $\mathrm{V} 2$ it is not; points above and below the horizontal meridian project to non-adjacent sets of cortical columns in this area. In this and all subsequent figures, the visual hemifield is illustrated from the experimenter's point of view (see top right of panel C).

\footnotetext{
${ }^{1}$ The vertical and horizontal meridians are the axes of reference usually employed in describing visuotopic organisations. The vertical meridian is an imaginary vertical line through the fixation point, which subdivides the visual field into right and left hemifields. It corresponds to the line of decussation between the left and right hemiretinae. The horizontal meridian is a line perpendicular to the vertical meridian, through the fixation point; it subdivides each hemifield into an upper and a lower quadrant.
} 


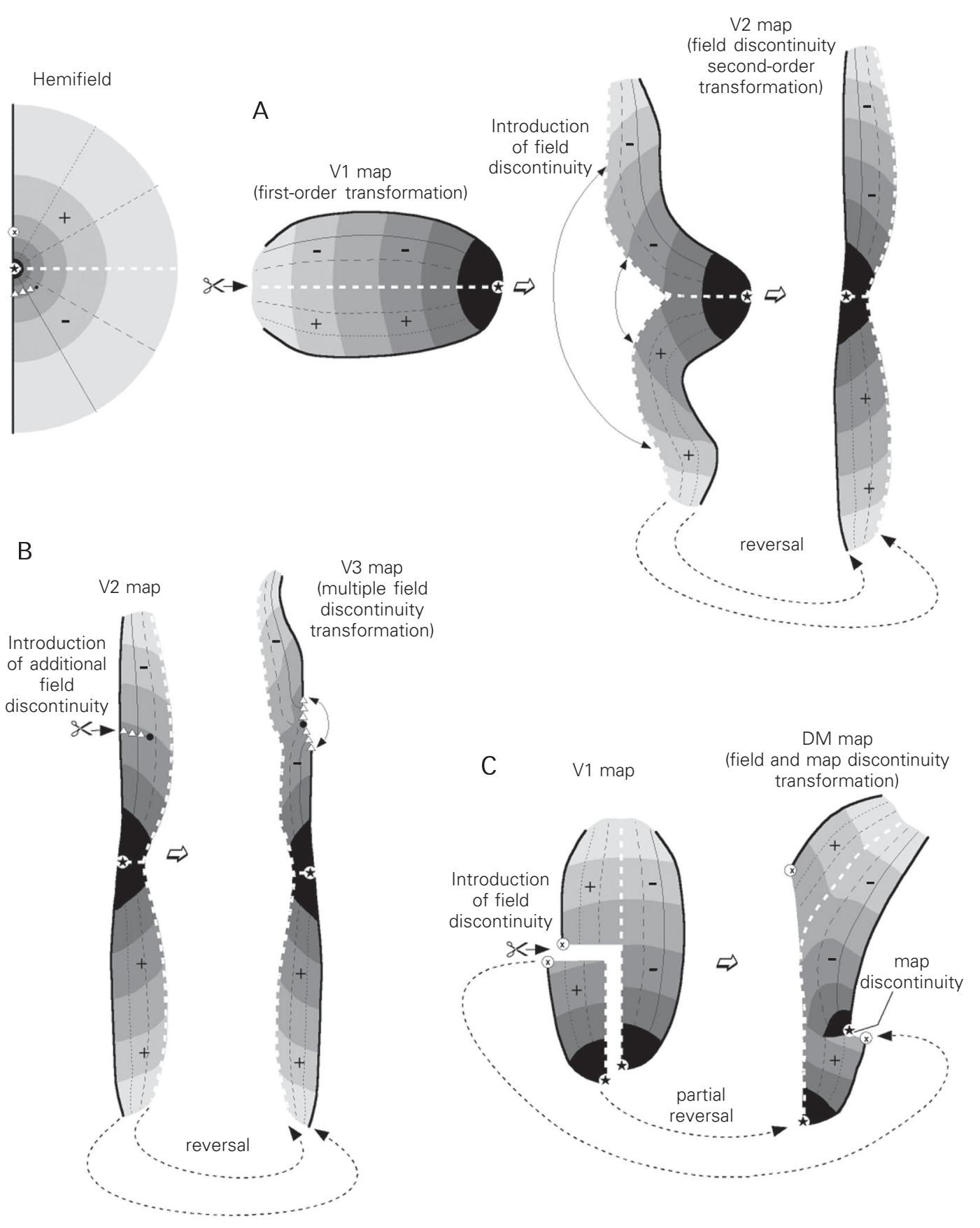

Figure 2. Different types of visuotopic map can be seen as topological transformations of the $\mathrm{V} 1$ map. Top left, Schematic representation of the visual hemifield, illustrating the coding of different visual field parts used in the maps shown in A-C. A, "Simple" field discontinuity maps, such as V2, can be imagined as a result of cutting and physically separating a first-order representation map. B, Visuotopic maps can include secondary field discontinuities, such as those observed along the rostral border of primate $\mathrm{V} 3$ (circle and triangles). $C$, More rarely, maps may include map discontinuities, regions where the receptive field coordinates change suddenly. For example, in the dorsomedial (DM) area of marmosets the central and peripheral parts of the upper contralateral hemifield are represented in spatially segregated sectors. This transformation can be imagined as the result of cutting off an entire "island" of cortex, and re-attaching it to the map with a different orientation. As a result, there is a small region along the rostral border of DM where cells with receptive fields in the mid-peripheral region of the upper quadrant adjoin cells with receptive fields in the central lower quadrant (map discontinuity). Note, however, that this transformation preserves the continuity and the centroperipheral axis of the horizontal meridian representation (white dashed line), resulting in a congruent border between DM and V2 (see also Figure 3). 
diately anterior to V2 (Figure 3). Based on early anatomical tracing studies, the organisation of this area in primates was originally considered to be a reduced mirror-image version of V2, with a continuous representation of the vertical meridian forming its anterior boundary $(13,20,21)$. However, subsequent detailed electrophysiological studies have revealed that several locations along the rostral boundary of $\mathrm{V} 3$ represent parts of the visual field away from both the vertical and the horizontal meridians, indicating that the visuotopic map includes multiple field discontinuities $(6,22)$. This type of transformation can be interpreted by imagining that the visuotopic map of V2 (which, as outlined above, already includes a "primary" field discontinuity) has received further cuts along its perimeter. The outcome of this (Figure $2 \mathrm{~B}$ ) is that the rostral boundary of V3 is formed in part by cells with receptive fields including the vertical meridian, and in part by cells representing other sectors of the visual field. The entire vertical meridian is still represented, but in a discontinuous manner, such that the representation is located in two mutually complementary regions of the cortex. This complex topography of V3 has been observed in all three species of monkey that have been extensively studied by means of electrophysiological recordings $(6,22,23)$. Multiple field discontinuities also appear to exist in the middle temporal crescent (MTc; this area includes the "V4 transitional" region), where the representation of the horizontal meridian receives further "cuts". Thus, the receptive fields of cells near the outer boundary of this area sometimes represent the horizontal meridian, and sometimes other parts of the visual field (10). Finally, the pattern of secondary field discontinuities may differ significantly between individuals of the same species. Although this has been best documented in carnivores (24-27), suggestions of similar variability have arisen in primates $(6,22)$.

In the areas described above, adjacent cortical columns always represent adjacent or overlapping parts of the visual field. While this is characteristic of the great majority of cortical representations, there are exceptions. For example, a map discontinuity (i.e., an imaginary line separating two adjacent populations of neurones with non-overlapping receptive fields) exists in primary somatosensory cortex, at the boundary between the representations of the hand and the face (28). To date, it remains unclear whether cortical map discontinuities correspond to regions of extremely rapid change in receptive field position, or true "fractures" in the representation, where adjacent neurones can have non-overlapping receptive fields.

In primate visual cortex, the only welldocumented example of a second-order transformation including a map discontinuity is the dorsomedial (DM) area of marmosets (29). The visuotopic organisation of DM includes both a field and a map discontinuity (Figure 2C). As a result of the field discontinuity, the representation of the upper quadrant in this area occupies two non-adjacent regions: a lateral sector, which represents the central visual field, and a medial sector, which represents the peripheral visual field. In macaques, a similar pattern of representation has been revealed in visual area 6 (V6; 30 ), the Old World monkey homologue of DM (7). In both DM and V6, the medial sector of the upper quadrant representation is topologically continuous with the lower quadrant representation, creating a relatively simple map of the peripheral visual field (this peripheral representation is sometimes referred to as the "parietooccipital area"). However, at least in the marmoset (Figure $2 \mathrm{C}$ ), the lateral sector of upper quadrant representation adjoins the remainder of DM along a map discontinuity, which separates cells with receptive fields in parafoveal and mid-peripheral parts of the upper quadrant and cells with receptive fields in the foveal lower quadrant.

In visual areas located in the caudal part 


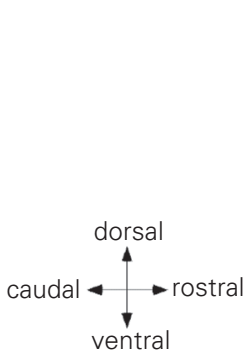

A

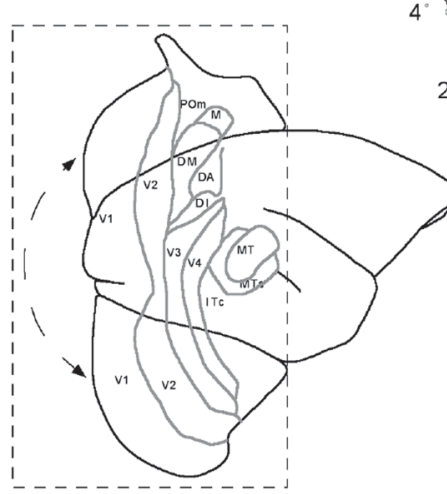

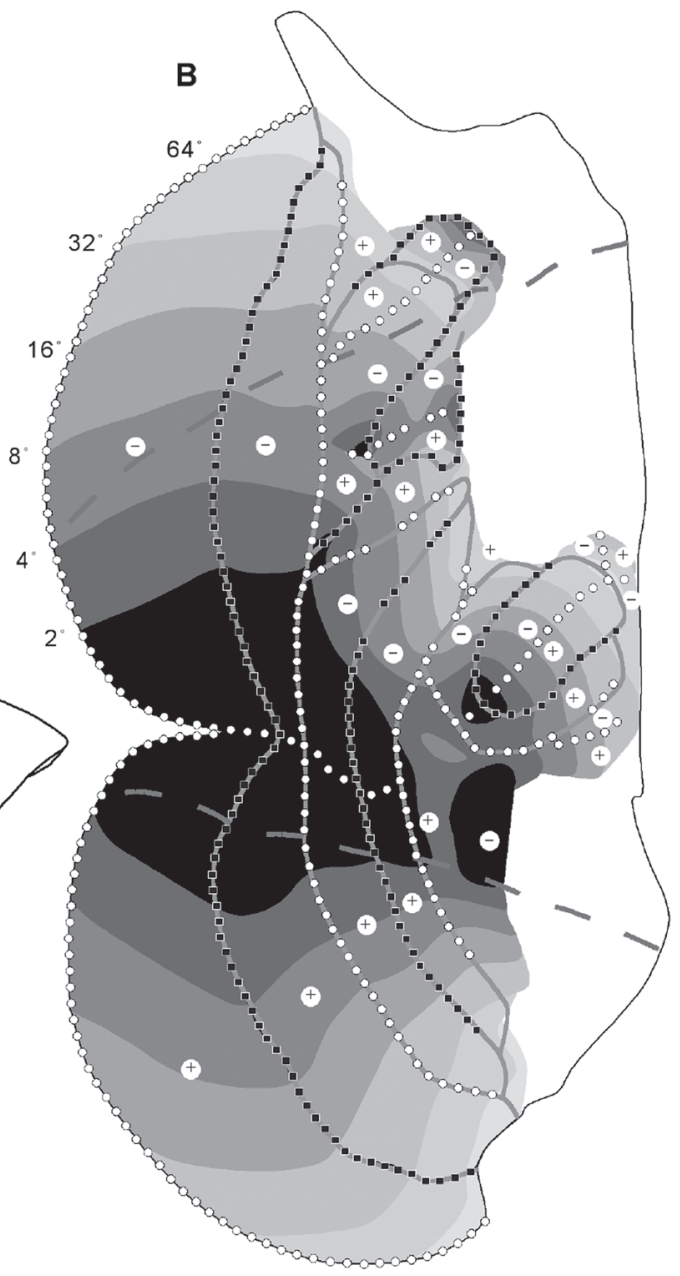

Figure 3. Supra-areal visuotopic organisation of primate visual cortex. A, Lateral view of the right hemisphere of a marmoset monkey, illustrating the anatomical relationships within the cortical region shown in part B. In generating this figure parts of the cerebral cortex normally hidden from view (those located along the dorsal midline and ventral surface) have been graphically "unfolded"; moreover, an artificial discontinuity has been introduced along the horizontal meridian representation in V1 (arrows). The grey lines indicate the boundaries of cortical areas, according to a scheme of subdivision based on physiological mapping and architecture $(6,10,29)$. $B$, Magnified view of the same map, with the visual topography indicated. The thick dashed lines indicate the dorsal and ventral limits of the cortex that is normally exposed on the dorsal and lateral surfaces of the brain. The numbers to the left of $\mathrm{V} 1$ indicate the range of receptive field centre eccentricities observed within the regions coded by the different tones of grey $\left(0-2^{\circ}, 2-4^{\circ}, 4-8^{\circ}\right.$, etc. $)$. Representations of the horizontal meridian are indicated by white circles, representations of the vertical meridian by black squares, representations of the upper quadrant by the "+" signs, and those of the lower quadrant by the "-" signs. Although it is likely that visuotopy extends beyond the areas shown in this figure, the detailed organisation of these maps has not yet been established in the marmoset. POm, parietooccipital medial area; M, medial visual area; DM, dorsomedial area; DA, dorsoanterior visual area; DI, dorsointermediate visual area; MT, middle temporal visual area; MTc, middle temporal crescent; ITc, caudal subdivision of the inferior temporal visual area.

of the brain, including V1 and most of the areas connected to it, cells are maximally responsive to stimuli presented within relatively well-defined receptive fields. By contrast, in areas near the occipitotemporal and occipitoparietal transitions the single unit receptive fields are large, and the visuotopic organisations are best described in terms of coarse gradients. These rostral visual areas cannot be appropriately described in terms of the transformations discussed above. For example, multiunit recordings in cytoarchitectural area TEO have revealed a gradual change of receptive field locations from the central to the peripheral visual field, and a segregation between cells representing the upper and lower contralateral quadrants $(6,31)$. However, despite these general trends, changes in receptive field position cannot be predicted with any accuracy, and their centres alternate between the vicinities of the horizontal and vertical meridians in a seemingly random manner. Due to this large scatter, a continuous representation of each quadrant is still achieved, without a systematic mapping of polar angle. Other regions that preserve a coarse segregation between the representations of the upper, lower, central and peripheral visual fields include visual area $6 \mathrm{~A}(\mathrm{~V} 6 \mathrm{~A} ; 32)$, the lateral intraparietal area (33), and the motion-sensitive areas adjacent to MT (34). In the region of the inferior temporal cortex located in the ventral bank of the superior temporal sulcus, only a crude centroperipheral gradient is preserved; cells with receptive fields centred in the upper and lower quadrants are observed in no particular order (35).

A last possible category of visuotopic map is that of incomplete representations. A number of studies have raised the possibility of maps which fail to represent all regions of the visual field. However, more extensive examination often reveals that areas with supposedly incomplete maps are actually parts of larger areas with complete visual field representation. One such case, the 
"parietooccipital area", was initially described as having no foveal representation, but can now be viewed as the peripheral representation of a "complete" area, V6 $(7,30)$. Similarly, the "ventral posterior area", which was once characterised as representing only the upper quadrant of the visual field, is now known to correspond to the ventral half of a larger subdivision, which includes near-symmetrical representations of both quadrants (6). At present, there is no compelling evidence for areas in the primate cortex with grossly incomplete representations. A related, but more difficult problem is determining whether or not there are visual areas lacking representations of the far periphery of the visual field. In most areas, the major emphasis on representation of central visual fields creates significant sampling problems, making it difficult to determine on the basis of microelectrode recordings alone whether far peripheral fields are truly absent from a map.

\section{Supra-areal visuotopic organisation}

The above descriptions of visuotopic maps fail to convey one of the most striking features of the primate visual cortex: the coherence of visual topography across functional boundaries. Receptive field position does not change suddenly as the boundary between two areas is crossed. Instead, the gradient of representation usually reverts in such a way that adjacent maps are mirrorsymmetrical in the neighbourhood of their common boundary. Less frequently, the representations of the upper and lower quadrants in two adjacent areas meet at a border that represents the horizontal meridian. The continuity of visuotopic organisation is clearly demonstrated by Figure 3, which presents a composite view of the visuotopic organisations of several areas in the marmoset monkey. For example, the foveal representations of several areas align near the lateral convexity of the occipital lobe, form- ing an elongated strip that does not respect areal boundaries. Similarly, the representations of gradually more eccentric parts of the visual field form continuous bands, which occupy progressively more medial locations. Although neurones near areal boundaries often have receptive fields which include the vertical and horizontal meridians, this is not necessarily the case, as outlined above.

\section{Summary: the main rules of cortical visuotopic organisation}

In conclusion, there appear to be two underlying rules which govern the topology of cortical visuotopic maps and which may be significant in determining the mechanisms involved in the formation of cortical areas.

Rule 1: adjacent radial columns in the cortex must represent adjacent regions of the visual field, irrespective of whether these columns are part of the same or adjacent areas. It remains unclear whether or not there are any exceptions to this rule (true map discontinuities, where adjacent cell columns have non-overlapping receptive fields). At the microscopic level, map discontinuities may be bridged by neurones with rapidly changing (23), unusually large (36), or dual (Calford MB and Rosa MGP, unpublished observations) receptive fields.

Rule 2: adjacent regions of the visual field tend to be represented in adjacent radial columns of a same given area. This rule is not as strictly "enforced" as the first one. For example, second-order representations of the visual field are far more common than first-order representations.

\section{Implications for developmental mechanisms guiding the formation of cortical maps}

Despite a significant body of theoretical and modelling studies, there has been relatively little experimental work on the forma- 
tion of cortical visuotopic maps (37). Moreover, it is only recently that the genetic mechanisms underlying the specification of cortical areas have begun to be elucidated $(38,39)$. Thus, it may be instructive at this point to consider the implications which the characteristics of adult maps, reviewed above, may have for understanding cortical development.

Current views regarding the formation of visuotopic maps are strongly influenced by studies on retino-recipient subcortical nuclei, such as the superior colliculus (40). In these projections, gradients of cell surface chemicals define the polarity of the representation by guiding afferent axons to their approximate targets. This genetically "hardwired" step determines the coarse features of the map, such as the orientation of the centro-peripheral axis. An activity-dependent stage follows once afferents establish synaptic contact, and the map is gradually refined. Simulations of activity-dependent processes in spatially contained structures (e.g., nuclei with sharp histological limits) have indicated that first-order representations can form spontaneously, requiring only temporally patterned inputs and Hebbian rules (41). Given that retinal adjacency is such a powerful determinant of correlated activity (42), it is biologically plausible that a precise map reflecting the receptor densities would form in these structures, even if only on the basis of the epigenetic, activitydriven step (43). The initial guidance provided by cell membrane ligands provides a nonrandom starting point for the activity-dependent stage, which helps to explain consistency of the maps between individuals.

The fact that visuotopy is relatively consistent across individuals suggests that a chemical specification step may also characterise the initial stages of cortical map formation, although the evidence in visual cortex remains inconclusive and alternative mechanisms have been proposed (44). In addition, there is substantial evidence to sug- gest that an activity-dependent component is involved in the formation of cortical maps (e.g., 45). Despite these parallels, there are at least two important characteristics of cortical areas that need to be accounted for by any model of cortical map development. First, the boundaries of cortical areas are rarely sharply defined; most do not coincide with obvious architectural transitions, there being no evidence of sharp interruptions in columnar patterns or intrinsic connections across borders $(46,47)$. Rather, cortical areas could be viewed as gradually merging into each other. Second, as outlined above, overlap between the receptive fields of cells in adjacent columns in adult cortex takes precedence over continuity in the representation within a given area. This local congruency of receptive field topography is maximised both within and across areal boundaries. These observations suggest that visual cortical maps are not "independent" of each other, and that it may not be appropriate to think in terms of the development of each area separately.

One possibility is that the characteristics of each cortical map are specified independently, according to the same mechanisms which are known to drive the genesis of subcortical maps: "seeding", early in development, by gradients of cell surface signalling molecules, followed by synaptically dependent functional interactions. Support for this hypothesis comes from studies of the primary somatosensory area, where specification of the mediolateral axis of representation appears to be directly related to a graded distribution of Ephrin-A5 (48). The interareal congruency of topography in the adult cortex could be a consequence of gradients of cell surface markers which crossed the boundaries of developing areas; for example, a band-like distribution indicating the location of a vertical meridian representation could be "shared" across two future areas. This compares with the more demanding alternative of specifying visual topographies 
with independent molecular gradients for each developing area. However, one problem with the molecular specification hypothesis lies in the complex distribution pattern of cell surface molecules required to achieve the large number of areas and the prevalence of second-order representations seen in visual cortex. While the outlines of first-order representations can be specified on the basis of only two monotonic gradients of cell surface molecules (e.g., centroperipheral and dorsoventral), the specification of secondorder representations on the basis of afferents arising from a first-order map would require more complicated, and non-monotonic patterns of molecule distribution. For example, the maps of V2, V3 and V4 would require molecules specifying a putative foveal-peripheral gradient to distribute simultaneously from the middle of these areas to the dorsal and ventral extremities. The specification of polar angles would be even more complicated, perhaps requiring alternating, band-like distributions.

An alternative to this complex molecular pattern is to have a few key maps specified by molecular cues, and serving as topographic "anchors" for the development of adjacent areas; the visuotopy in these other areas would then be largely defined by activitydependent processes. Let us consider, for example, that the V1 map develops in a similar manner to the collicular map (i.e., broad genetic specification, chemical or otherwise, followed by refinement), and that the V2 map self-organises around it according to Hebbian rules imposed by correlated retinal activity. The need to preserve a congruent boundary with V1 would be sufficient condition to limit the range of possible V2 configurations to those observed experimentally in adult primates (e.g., cells near the posterior border of $\mathrm{V} 2$ would necessarily have receptive fields near the vertical meridian). This hypothesis predicts the existence of a type of cellular interaction during the activity-dependent stage, which limits adja-
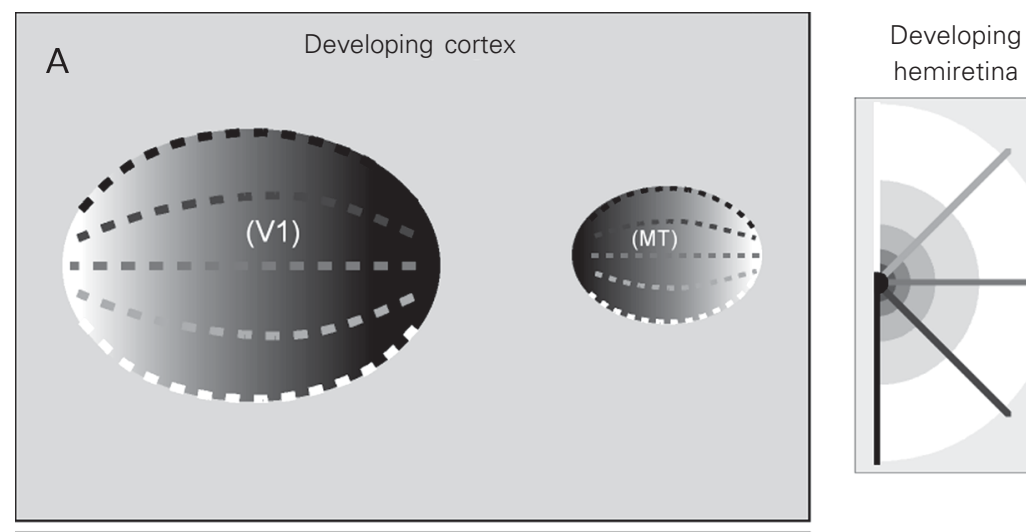

hemiretina
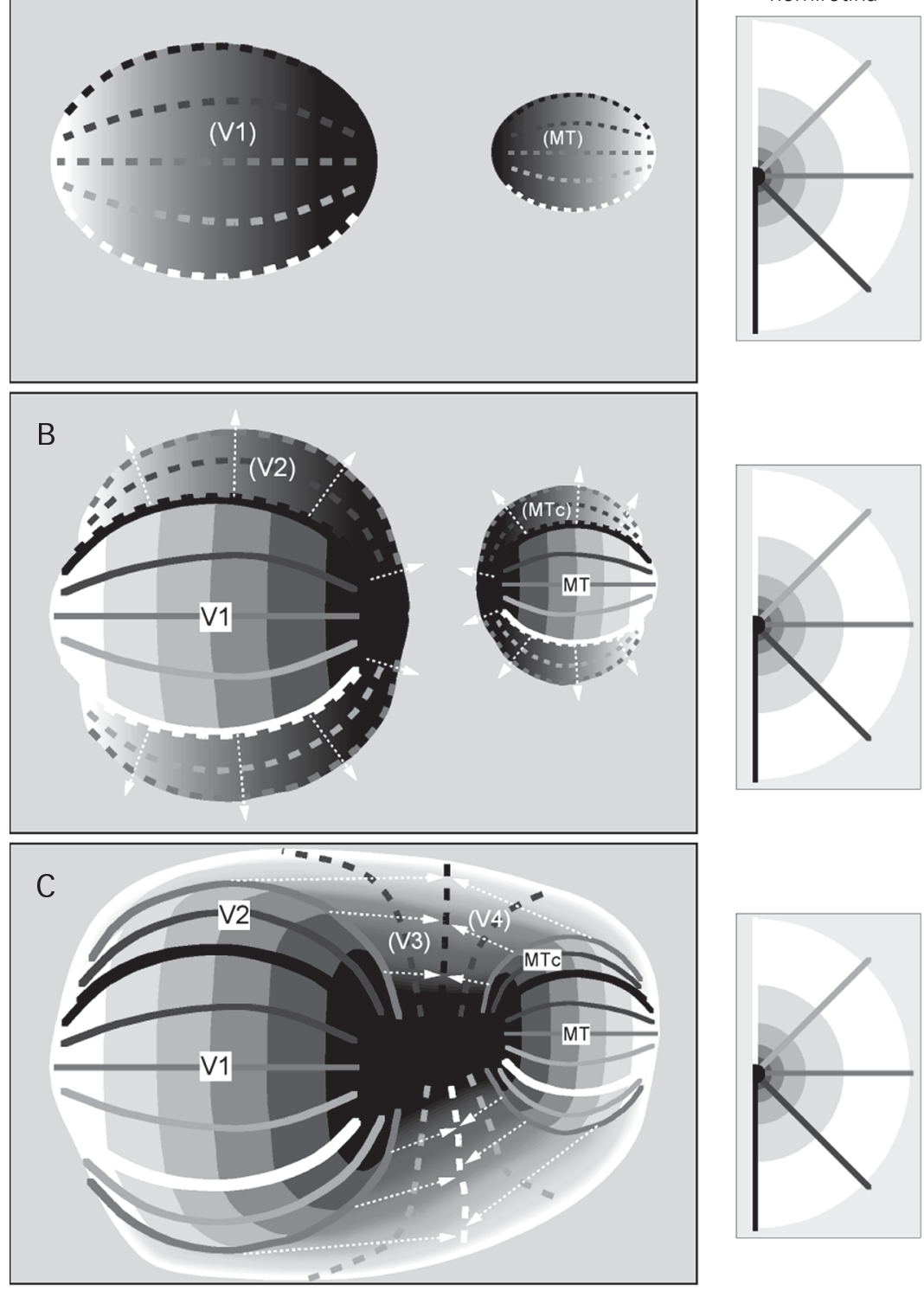

Figure 4. Schematic view of the development of visuotopic maps according to the "molecular anchors" hypothesis. In these diagrams, dashed lines and continuous grey scale gradients indicate regions undergoing early stages of the process of visuotopic map formation. A, Two primary visual maps (corresponding to adult visual primary (V1) and middle temporal (MT) areas, the only first-order representations in the adult brain) are specified early in development, either by gradual distributions of cell surface chemoattractant/chemorepellent molecules (40) or by spatiotemporal patterning of the afferent projection (44). B, With the V1 and MT maps defined, the visuotopic maps in adjacent areas (V2 and MTc) start to self-organise around these "anchors". Two rules guide this process: 1) the receptive fields of neurones in adjacent columns must overlap, and 2) the gradient of representation does not revert within a given area (arrows). This ensures that the same part of the visual field is not represented more than once in a given area, except along its boundaries. C, At a later stage of development, additional maps (e.g., V3 and V4) selforganise, using the established maps of V2 and MTc as "anchors". Throughout pre- and post-natal development, activity-dependent mechanisms allow the fine-tuning of the maps. 
cent cells to having receptive fields in the same part of the visual field (a biological implementation of "rule 1" seen above). Such an interaction would occur throughout the early developing visual cortex, both within and across the borders of future areas (possibly involving the extensive network of interneuronal gap junctions observed during this period; 49). In such a scenario, those areas whose visuotopic identity is specified early in development could progressively limit the options governing the creation of subsequent maps. In fact, a simple model invoking the

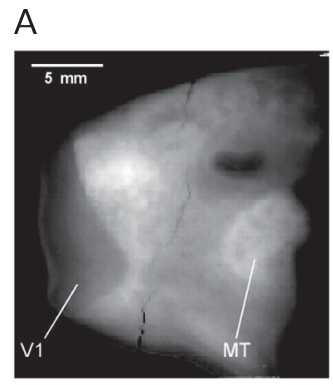

B
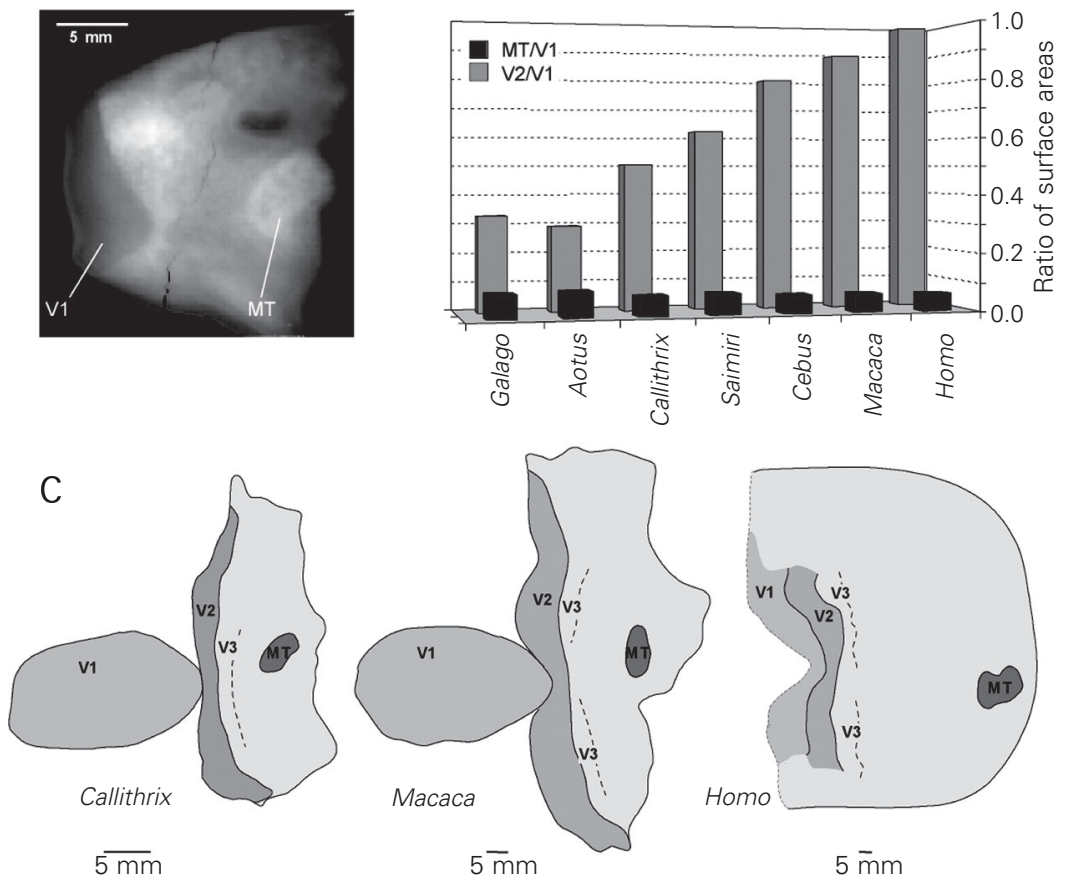

Figure 5. A, Flat-mounted vibratome slice through the brain of a marmoset. Caudal is towards the left, and dorsal towards the top. This slice has not been stained, and the borders of primary visual (V1) and middle temporal (MT) areas are made visible only by differences in myelination (highly myelinated areas appearing lighter). Although the rostral border of $\mathrm{V} 2$ is only faintly visible in this type of preparation, it can be easily defined in cytochrome oxidase-stained material. B, Surface areas of V2 (light grey) and MT (dark grey) as a fraction of the V1 surface, in seven species of primates. All measurements were obtained from flat-mounted sections stained for myelin or cytochrome oxidase. C, Graphically "unfolded" reconstructions of the posterior neocortex of three species of diurnal primates with different brain sizes (note different scale bars at the bottom of each panel). The extents of V1, V2 and MT, as well as the location of rostral border of V3, are indicated. Left: marmoset; middle: macaque; right: human (from Ref. 53). In order to reduce the distortions introduced by the graphic unfolding, discontinuities were introduced in the maps, either along the perimeter of V1 (left, centre) or across this area (right). The human map does not indicate the full extent of V1 and V2, as only the central $15-20^{\circ}$ of the visual field were mapped, using non-invasive imaging techniques. existence of two first-order representation "anchors" (V1 and MT) and time-dependent gradients of map maturation is sufficient to explain many of the characteristics of visuotopic maps observed in adult primate cortex (Figure 4). As argued below, several lines of evidence suggest that V1 and MT may be both regarded as "primary" visual areas, in functional and evolutionary terms. It has also been demonstrated that they mature earlier than adjacent areas in terms of characteristics such as myelination and distribution of interneurones $(50,51)$.

The molecular anchors hypothesis requires some cellular signal at the borders of late developing representations, to mark the point at which representational gradients should stop progressing. For example, while the receptive fields of neurones forming dorsal V2 could be expected to self-organise from the vertical to the horizontal meridian in the lower field (Figure 4B), it is more difficult to explain why the map stops at the horizontal meridian, instead of invading the upper visual quadrant. One possibility is that the future borders of areas are pre-specified by molecular cues (38), and that one of the functions of the observed transitions in molecular "identity" patterns is to signal the points where reversals in the representational gradients occur.

Finally, as a third possibility, it has been proposed that the early stages of visuotopic map formation in the cortex are completely dependent on the spatial organisation of thalamic maps. For example, according to one point of view, the visuotopic organisation of the pulvinar complex replicates in detail that of the extrastriate cortex (52). Thus, the assignment of visuotopic identities to cortical cells could be accomplished by mechanisms such as the preservation of adjacency relationships in the thalamocortical bundles, spatiotemporally graded maturation of the thalamocortical circuitry (44), or corresponding monotonic gradients of molecules. Unfortunately, the merit of this hypothesis re- 
mains difficult to judge, as the topographic organisation of the pulvinar nuclei is still a subject of intense debate. However, even if the assumption of isomorphic cortical and thalamic maps proves to be true, this would merely shift the emphasis to explaining the formation of different types of thalamic visuotopic representations. The same issues raised above (complexity of the required molecular gradients, mechanism for preserving congruency) would still apply.

\section{Primate visual cortex in evolution}

Whatever mechanisms are responsible for the establishment of visuotopic maps in the developing cortex, it is clear that they allow for considerable variation among species. Thus, although much can be learned about the principles of organisation of the human visual system from studies in monkeys, the proper interpretation of data also requires knowledge about the rules governing this variability. Moreover, a long-term evolutionary perspective can be helpful in interpreting mechanisms of brain development, by demonstrating which types of changes are likely to occur, and which of these are unlikely to succeed in the context of natural selection.

Larger primate brains are not simply homogeneously scaled versions of smaller brains. This can be demonstrated by comparing the relative sizes of clearly defined cortical fields, such as V1, V2 and MT, which can be directly visualised in histological sections (Figure 5A,B). Despite a fourteen-fold change in absolute values between marmosets and humans, the ratio of the surface areas of MT and V1 shows only a modest variation among primates (MT occupying cortex equivalent to $6-8 \%$ of the surface area of V1). In contrast, the relative size of V2 changes dramatically, from being nearly equal to that of $\mathrm{V} 1$ in larger primates with diurnal habits (Homo, Macaca) to about one-third of the size, in nocturnal primates
(Aotus, Galago). Smaller diurnal New World species lie between these extremes. The expansion of V2 in larger primate species has been accompanied by a relative growth of the visual cortex situated between V2 and MT, including the third and fourth visual complexes (Figure 5C). In humans, this region also includes what appear to be evolutionarily "new" areas, which have no clear homologues in the most intensively studied species of monkey $(53,54)$. These areas, located rostral to the putative homologue of V4, are characterised by ill-defined visuotopic organisations, including extensive representations of the ipsilateral hemifield. Conversely, in flying foxes (animals which may share some of the cortical organisation present in early primates), there is only a narrow strip of cortex interposed between V2 and MT, which is entirely devoted to a single, V3-like topographically organised area (55).

These observations suggest that one of the main trends in the evolutionary history of anthropoids has been the selective expansion of the visual cortex between V1 and MT. This can be interpreted in the light of the molecular anchors hypothesis (Figure 4). An early origin of these areas (both in terms of evolution and development) could be related to characteristics such as the presence of first-order representations of the visual field and sharp architectural boundaries. Newer areas, in contrast, have more complex visuotopic maps, less defined visual topographies, and are architecturally indistinct. It is intriguing that V1 and MT also share a number of important functional characteristics, including being cortical targets of thalamic retino-recipient nuclei (56), and responding to visual stimulation in parallel (57). In fact, it could be argued that they are both "primary visual areas".

\section{On the relationship between visuotopic maps and areas}

The current predominant view is that the 
cerebral cortex is divided into morphologically distinct, functionally dedicated and stereotypically connected fields, reflecting Brodmann's view of areas as the "organs of the brain". However, it should be realised that our knowledge of cortical organisation is far from being this clear. Even in the most intensively studied animal models, the degree of precision and confidence with which one can delimit visual areas varies enormously, from pencil-sharp limits to tenuous transitions. There are relatively few examples of well-segregated areas (mainly, primary sensory/motor areas), and it is possible that intermediate configurations, including partially overlapping or interdigitating fields, exist. This is clearly illustrated by what we know of visual areas. There are some, such as V1, which can be unambiguously identified using a number of different techniques, while other regions, despite intensive investigation, are still not well characterised. For example, histological transitions in the inferior temporal cortex are gradual, the patterns of interconnections are difficult to assign as clearly "intrinsic" or "extrinsic" $(58,59)$, and visuotopic maps are coarse. All evidence considered, the organisation of this region

Figure 6. Alternative hypotheses regarding the organisation of the third visual complex. Lateral views of the brain of a New World monkey, illustrating two models of the organisation of the cortex immediately anterior to $\mathrm{V} 2$. These are graphically "unfolded" views of the cortex hidden in the medial
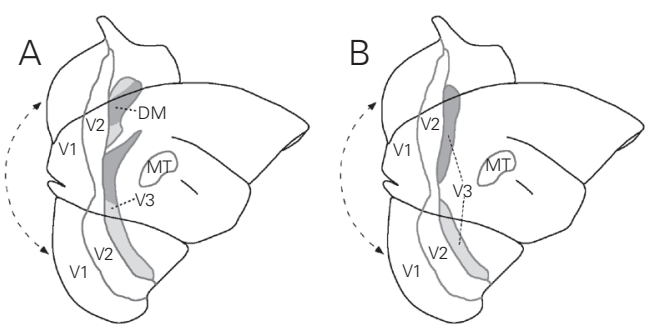
and ventral surfaces of the brain.

Light grey indicates representations of the upper contralateral quadrant (or parts thereof), and dark grey indicates representations of the lower contralateral quadrant. A, Hypothesis based on the results of electrophysiological recordings. According to this view, the dorsolateral and ventral regions of extrastriate cortex include a field homologous to area 19 of most mammals, named the ventrolateral posterior area (VLP), or V3 (6). Because neurones with receptive fields in the upper quadrant have been recorded immediately anterior to V2 in the dorsomedial cortex, the extension of $\mathrm{V} 3$ to the dorsal midline is regarded as unlikely. Instead, it is proposed that a dorsomedial area (DM) exists in this region. DM and V3 also differ in terms of myeloarchitecture. $B$, An alternative hypothesis, based on the results of anatomical tracing (60). As in the first diagram, the cortex anterior to ventral V2 forms a relatively simple representation of the upper quadrant. However, it is hypothesised that the dorsomedial cortex anterior to dorsal V2 lacks upper quadrant representations. Instead, it is proposed that a simple strip-like dorsal $\mathrm{V} 3$ exists in the corresponding location, representing only the lower contralateral quadrant. V1, V2, MT: primary, second and middle temporal visual areas. can be just as adequately described in terms of gradual changes, without the implication of sharply defined, functionally segregated areas.

Falling between these extremes lie a number of topographically organised visual areas which remain a source of controversy. One such example concerns the organisation of the cortical belt immediately anterior to V2 in monkeys (Figure 6). Although it has become now clear that "ventral V3", or the ventral posterior area, is part of a larger area which represents both the upper and lower quadrants (6), studies regarding the extent and visuotopy of dorsal V3 (the lower quadrant representation) have generated incompatible results. According to one interpretation, based on electrophysiological data in New World monkeys, there is a cortical area (DM) representing the upper quadrant immediately adjacent to dorsal V2 (Figure 6, left), and the V3-like area is restricted to ventral and lateral cortices. However, anatomical tracing experiments in the same species have given rise to a different interpretation (Figure 6, right), suggesting that dorsal $\mathrm{V} 3$ in fact extends to the dorsal midline, separating V2 from DM (60).

Although many of the current controversies may be solved by future studies, one must also be prepared to accept the possibility that the cerebral cortex is not a mosaic of clearly defined, homogeneous fields. Borders which are sharply defined and methodologically robust in adults may well reflect those which are specified by the sharpest transitions in the expression of cell surface molecules during development (e.g., V1;38). As discussed earlier, such boundaries are likely to be characteristic of phylogenetically older areas, which change relatively little across primate species. In evolutionarily newer cortices, interdigitating areas, gradual transition zones, more complex visuotopic maps and larger individual variability are to be expected, particularly if activ- 
ity-dependent processes respond for a large fraction of the formation of the circuitry. These regions are also likely to be more variable among species, thereby compounding the inherent difficulty in identifying homologous fields (54). Clarification of the developmental mechanisms underlying the formation of cortical maps, and in particular the degree of genetic specification involved in the establishment of different areas, will have a significant impact on our understanding of the functional organisation and evolu- tion of the visual cortex.

\section{Acknowledgments}

Equipment support from the Clive and Vera Ramaciotti Foundation and the ANZ Charitable Trust is gratefully acknowledged. Special thanks to Prof. Roberto Lent, for the organisation of a fantastic symposium, and to Rowan Tweedale, for many hours of discussion and for reading earlier versions of this paper.

\section{References}

1. Hubel DH \& Wiesel TN (1974). Uniformity of monkey striate cortex: A parallel relationship between field size, scatter, and magnification factor. Journal of Comparative Neurology, 158: 295-306.

2. Cowey A (1964). Projection of the retina onto striate and prestriate cortex in the squirrel monkey, Saimiri sciureus. Journal of Neurophysiology, 27: 366-393.

3. Allman JM \& Kaas JH (1971). Representation of the visual field in striate and adjoining cortex of the owl monkey (Aotus trivirgatus). Brain Research, 35: 89-106.

4. Allman JM \& Kaas JH (1974). The organization of the second visual area (V II) in the owl monkey: A second order transformation of the visual hemifield. Brain Research, 76: 247-265.

5. Allman JM \& Kaas JH (1975). The dorsomedial cortical visual area: A third tier area in the occipital lobe of the owl monkey (Aotus trivirgatus). Brain Research, 100: 473-487.

6. Rosa MGP \& Tweedale R (2000). Visual areas in lateral and ventral extrastriate cortices of the marmoset monkey. Journal of Comparative Neurology, 422: 621651.

7. Rosa MGP \& Tweedale R (2001). The dorsomedial visual areas in New World and Old World monkeys: homology and function. European Journal of Neuroscience, 13: $421-427$

8. Azzopardi P \& Cowey A (1993). Preferential representation of the fovea in the primary visual cortex. Nature, 361: 719-721.

9. Rosa MG, Fritsches KA \& Elston GN (1997). The second visual area in the marmoset monkey: visuotopic organisation, magnification factors, architectonical boundaries, and modularity. Journal of
Comparative Neurology, 387: 547-567.

10. Rosa MGP \& Elston GN (1998). Visuotopic organisation and neuronal response selectivity for direction of motion in visual areas of the caudal temporal lobe of the marmoset monkey (Callithrix jacchus): middle temporal area, middle temporal crescent, and surrounding cortex. Journal of Comparative Neurology, 393: 505-527.

11. Allman JM \& Kaas JH (1971). A representation of the visual field in the caudal third of the middle temporal gyrus of the owl monkey (Aotus trivirgatus). Brain Research, 31: 85-105.

12. Fritsches KA \& Rosa MGP (1996). Visuotopic organisation of striate cortex in the marmoset monkey (Callithrix jacchus). Journal of Comparative Neurology, 372: 264-282.

13. Zeki SM (1969). Representation of central visual fields in prestriate cortex of monkey. Brain Research, 14: 271-291.

14. Rosa MGP, Sousa APB \& Gattass $R$ (1988). Representation of the visual field in the second visual area in the Cebus monkey. Journal of Comparative Neurology, 275: 326-345.

15. Sereno MI, Dale AM, Reppas JB, Kwong KK, Belliveau JW, Brady TJ, Rosen BR \& Tootell RB (1995). Borders of multiple visual areas in humans revealed by functional magnetic resonance imaging. Science, 268: 889-893.

16. Rosa MGP, Casagrande VA, Preuss $T$ \& Kaas JH (1997). Visual field representation in striate and prestriate cortices of a prosimian primate (Galago garnetti). Journal of Neurophysiology, 77: 3193-3217.

17. Rosa MGP \& Krubitzer LA (1999). The evolution of visual cortex: where is V2? Trends in Neurosciences, 22: 242-248.
18. Allman JM \& Kaas JH (1974). A crescentshaped cortical visual area surrounding the middle temporal area (MT) in the owl monkey (Aotus trivirgatus). Brain Research, 81: 199-213.

19. Allman JM \& Kaas JH (1976). Representation of the visual field on the medial wall of occipital-parietal cortex in the owl monkey. Science, 191: 572-575.

20. Zeki SM (1977). Simultaneous anatomical demonstration of the representation of the vertical and horizontal meridians in areas $\mathrm{V} 2$ and $\mathrm{V} 3$ of rhesus monkey visual cortex. Proceedings of the Royal Society of London. B, Biological Sciences, 195: 517-523.

21. Cragg BG \& Ainsworth A (1969). The topography of the afferent projections in the circumstriate visual cortex of the monkey studied by the Nauta method. Vision Research, 9: 733-747.

22. Gattass R, Sousa APB \& Gross CG (1988). Visuotopic organization and extent of $\mathrm{V} 3$ and $\mathrm{V} 4$ of the macaque. Journal of Neuroscience, 8: 1831-1845.

23. Sereno MI, McDonald CT \& Allman JM (1994). Analysis of retinotopic maps in extrastriate cortex. Cerebral Cortex, 4: 601-620.

24. Tusa RJ, Rosenquist $A C$ \& Palmer LA (1979). Retinotopic organization of areas 18 and 19 in the cat. Journal of Comparative Neurology, 185: 657-678.

25. Albus K \& Beckmann R (1980). Second and third visual areas of the cat: interindividual variability in retinotopic arrangement and cortical location. Journal of Physiology, 299: 247-276.

26. Sherk H \& Mulligan KA (1993). A reassessment of the lower visual field map in striate-recipient lateral suprasylvian cor- 
tex. Visual Neuroscience, 10: 131-158.

27. Manger PR, Kiper D, Masiello I, Murillo L, Tettoni L, Hunyadi Z \& Innocenti GM (2002). The representation of the visual field in three extrastriate areas of the ferret (Mustela putorius) and the relationship of retinotopy and field boundaries to callosal connectivity. Cerebral Cortex, 12: 423-437.

28. Manger PR, Woods TM, Munoz A \& Jones EG (1997). Hand/face border as a limiting boundary in the body representation in monkey somatosensory cortex. Journal of Neuroscience, 17: 6338-6351.

29. Rosa MGP \& Schmid LM (1995). Visual areas in the dorsal and medial extrastriate cortices of the marmoset. Journal of Comparative Neurology, 359: 272-299.

30. Galletti C, Fattori P, Gamberini M \& Kutz DF (1999). The cortical visual area V6: brain location and visual topography. European Journal of Neuroscience, 11: 3922-3936.

31. Boussaoud D, Desimone R \& Ungerleider LG (1991). Visual topography of area TEO in the macaque. Journal of Comparative Neurology, 306: 554-575.

32. Galletti C, Fattori $P$, Kutz DF \& Gamberini $M$ (1999). Brain location and visual topography of cortical area V6A in the macaque monkey. European Journal of Neuroscience, 11: 575-582.

33. Ben Hamed S, Duhamel JR, Bremmer F \& Graf W (2001). Representation of the visual field in the lateral intraparietal area of macaque monkeys: a quantitative receptive field analysis. Experimental Brain Research, 140: 127-144.

34. Komatsu H \& Wurtz RH (1988). Relation of cortical areas MT and MST to pursuit eye movements. I. Localization and visual properties of neurons. Journal of Neurophysiology, 60: 580-603.

35. Hikosaka K (1998). Representation of foveal visual fields in the ventral bank of the superior temporal sulcus in the posterior inferotemporal cortex of the macaque monkey. Behavioural Brain Research, 96: 101-113.

36. Payne BR \& Siwek DF (1990). Receptive fields of neurons at the confluence of cerebral cortical areas 17, 18, 20a, and 20b in the cat. Visual Neuroscience, 4: 475479.

37. Krug K, Smith AL \& Thompson ID (1998). The development of topography in the hamster geniculo-cortical projection. Journal of Neuroscience, 18: 5766-5776.
38. Donoghue MJ \& Rakic P (1999). Molecular gradients and compartments in the embryonic primate cerebral cortex. Cerebral Cortex, 9: 586-600.

39. Rubenstein JLR, Anderson S, Shi L, Miyashita-Lin E, Bulfone A \& Hevner $R$ (1999). Genetic control of cortical regionalization and connectivity. Cerebral Cortex, 9: 524-532.

40. O'Leary DD, Yates PA \& McLaughlin T (1999). Molecular development of sensory maps: representing sights and smells in the brain. Cell, 96: 255-269.

41. Willshaw DJ \& Von der Malsburg C (1976). How patterned neural connections can be set up by self-organisation. Proceedings of the Royal Society of London. B, Biological Sciences, 194: 431-435.

42. Wong RO, Meister M \& Shatz CJ (1993). Transient period of correlated bursting activity during development of the mammalian retina. Neuron, 11: 923-938.

43. Elliott T \& Shadbolt NR (1999). A neurotrophic model of the development of the retinogeniculocortical pathway induced by spontaneous retinal waves. Journal of Neuroscience, 19: 7951-7970.

44. Molnár Z, Adams R \& Blakemore C (1998) Mechanisms underlying the early establishment of thalamocortical connections in the rat. Journal of Neuroscience, 18: 5723-5745.

45. Herrmann K \& Shatz CJ (1995). Blockade of action potential activity alters initial arborization of thalamic axons within cortical layer 4. Proceedings of the National Academy of Sciences, USA, 92: 1124411248.

46. Malach R, Schirman TD, Harel M, Tootell RB \& Malonek D (1997). Organization of intrinsic connections in owl monkey area MT. Cerebral Cortex, 7: 386-393.

47. White LE, Bosking WH \& Fitzpatrick D (2001). Consistent mapping of orientation preference across irregular functional domains in ferret visual cortex. Visual Neuroscience, 18: 65-76.

48. Vanderhaeghen $\mathrm{P}, \mathrm{Lu} \mathrm{Q}$, Prakash N, Frisen J, Walsh CA, Frostig RD \& Flanagan JG (2000). A mapping label required for normal scale of body representation in the cortex. Nature Neuroscience, 3: 358-365.

49. Bittman K, Becker DL, Cicirata F \& Parnavelas JG (2002). Connexin expression in homotypic and heterotypic cell coupling in the developing cerebral cortex. Journal of Comparative Neurology, 443: 201-212.

50. Watson JDG, Myers R, Frackowiak RSJ,
Hajnal JV, Woods RP, Mazziotta JC, Shipp S \& Zeki SM (1993). Area V5 of the human brain: Evidence from a combined study using positron emission tomography and magnetic resonance imaging. Cerebral Cortex, 3: 79-94.

51. Condé F, Lund JS \& Lewis DA (1996). The hierarchical development of monkey visual cortical regions as revealed by the maturation of parvalbumin-immunoreactive neurons. Developmental Brain Research, 96: 261-276.

52. Dick A, Kaske A \& Creutzfeldt OD (1991). Topographical and topological organization of the thalamocortical projection to the striate and prestriate cortex in the marmoset (Callithrix jacchus). Experimental Brain Research, 84: 233-253.

53. Tootell RB, Mendola JD, Hadjikhani NK, Liu AK \& Dale AM (1998). The representation of the ipsilateral visual field in human cerebral cortex. Proceedings of the $\mathrm{Na}$ tional Academy of Sciences, USA, 95: 818-824.

54. Tootell RBH \& Hadjikhani N (2001). Where is 'dorsal V4' in human visual cortex? Retinotopic, topographic and functional evidence. Cerebral Cortex, 11: 298-311.

55. Rosa MGP (1999). Topographic organisation of extrastriate areas in the flying fox: implications for the evolution of mammalian visual cortex. Journal of Comparative Neurology, 411: 503-523.

56. O'Brien BJ, Abel PL \& Olavarria JF (2001). The retinal input to calbindin-D28k-defined subdivisions in macaque inferior pulvinar. Neuroscience Letters, 312: 145148.

57. Ffytche DH, Guy CN \& Zeki S (1995). The parallel visual motion inputs into areas $\mathrm{V} 1$ and $V 5$ of human cerebral cortex. Brain, 118: $1375-1394$.

58. Kondo H, Hashikawa $T$, Tanaka K \& Jones EG (1994). Neurochemical gradient along the monkey occipito-temporal cortical pathway. NeuroReport, 5: 613-616.

59. Suzuki W, Saleem KS \& Tanaka K (2000). Divergent backward projections from the anterior part of the inferotemporal cortex (area TE) in the macaque. Journal of Comparative Neurology, 422: 206-228.

60. Lyon DC \& Kaas JH (2001). Connectional and architectonic evidence for dorsal and ventral V3, and dorsomedial area in marmoset monkeys. Journal of Neuroscience, 21: 249-261. 